\title{
THE EFFECT OF SPATIAL LOCATION ON THE DETECTION OF CHANGE
}

\author{
by \\ Alexander Theus \\ A thesis submitted to \\ the Faculty of Graduate and Postdoctoral Affairs \\ in partial fulfillment of the requirements for the degree of \\ Master of Arts \\ in \\ Psychology \\ Carleton University \\ Ottawa, Canada
}


Library and Archives Canada

Published Heritage Branch

395 Wellington Street Ottawa ON K1A ON4 Canada
Bibliothèque et

Archives Canada

Direction du

Patrimoine de l'édition

395 , rue Wellington

Ottawa ON K1A 0N4

Canada
Your file Votre référence

ISBN: 978-0-494-83104-5

Our file Notre référence

ISBN: 978-0-494-83104-5
NOTICE:

The author has granted a nonexclusive license allowing Library and Archives Canada to reproduce, publish, archive, preserve, conserve, communicate to the public by telecommunication or on the Internet, loan, distribute and sell theses worldwide, for commercial or noncommercial purposes, in microform, paper, electronic and/or any other formats.

The author retains copyright ownership and moral rights in this thesis. Neither the thesis nor substantial extracts from it may be printed or otherwise reproduced without the author's permission.

\section{AVIS:}

L'auteur a accordé une licence non exclusive permettant à la Bibliothèque et Archives Canada de reproduire, publier, archiver, sauvegarder, conserver, transmettre au public par télécommunication ou par l'Internet, prêter, distribuer et vendre des thèses partout dans le monde, à des fins commerciales ou autres, sur support microforme, papier, électronique et/ou autres formats.

L'auteur conserve la propriété du droit d'auteur et des droits moraux qui protège cette thèse. $\mathrm{Ni}$ la thèse ni des extraits substantiels de celle-ci ne doivent être imprimés ou autrement reproduits sans son autorisation.
In compliance with the Canadian Privacy Act some supporting forms may have been removed from this thesis.

While these forms may be included in the document page count, their removal does not represent any loss of content from the thesis.
Conformément à la loi canadienne sur la protection de la vie privée, quelques formulaires secondaires ont été enlevés de cette thèse.

Bien que ces formulaires aient inclus dans la pagination, il n'y aura aucun contenu manquant. 


\begin{abstract}
Participants completed a change detection task with alternating arrays of letters that overlapped spatially or shifted. Displays were separated by a short ( $80 \mathrm{~ms})$ or long (500 ms) inter-stimulus intervals (ISI). The relative contributions of top-down and bottom-up information were assessed by manipulating familiarity (letter orientation) and the size of changes. When the ISI was short, RTs and error rates were higher for shifting displays, while the effect of familiarity did not differ as a function of whether displays overlapped or shifted. When the ISI was long, RTs were higher for shifting displays and the familiarity effect was larger for shifting displays than overlapping displays. Results indicate that changes are more difficult in shifting displays than overlapping displays. Top-down information (familiarity) about displays can be used to compensate for this greater difficulty, however only if sufficient time is available to consolidate sufficient representations of displays in short-term memory.
\end{abstract}




\section{Acknowledgments}

This project could not have been completed if not for the enthusiasm and support from my family, friends and academic peers and mentors. I gratefully acknowledge my supervisor, Dr. Chris Herdman, and Dr. Matthew Brown for constant feedback, insight and encouragement throughout the entire process. I am very grateful for the feedback and motivation I've received from my long-time collaborator, Dr. Mark Tovey. I would like to thank the members of my committee for their time and feedback which has helped to shape this thesis. I would also like to acknowledge the members of the ACE lab at Carleton University for the excellent work environment that they create.

I'd like to thank my Parents, Dan Theus and Julie Wilson, as well as my grandparents, Otto Theus, Maria Theus and Sheila Wilson for their unconditional support, which is the strongest foundation for any endeavour. 


\section{Page}

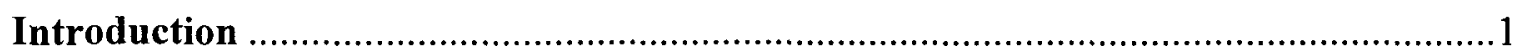

Spatial Location and Iconic Memory ….............................................

Spatial Location and Short-Term Memory ..........................................6

Conceptualizing Bottom-Up and Top-Down Processing ..........................11

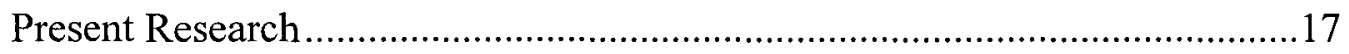

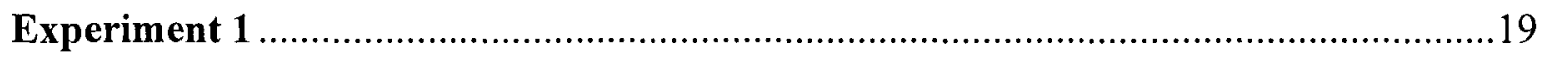

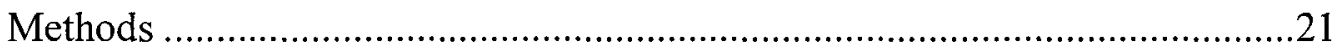

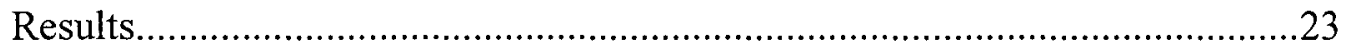

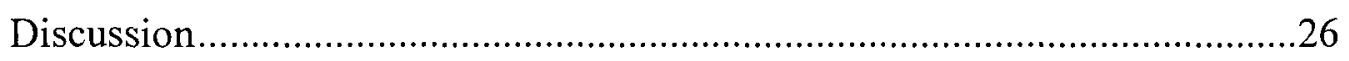

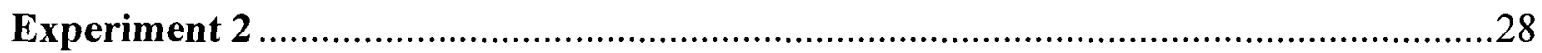

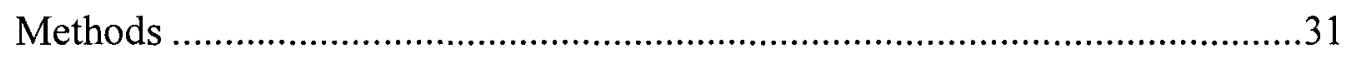

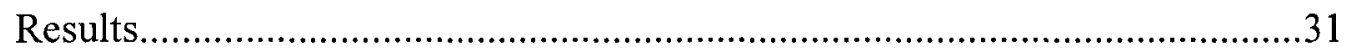

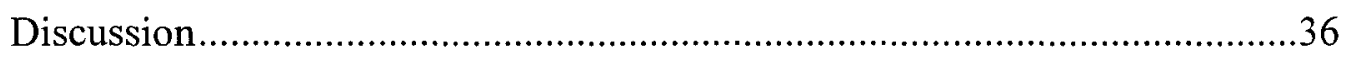

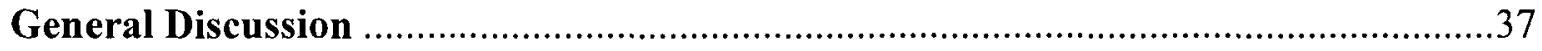

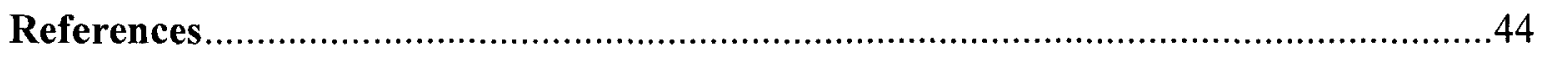




\section{List of Figures}

Figure 1. Diagram of iconic memory and short-term memory ................................

Figure 2. Upright and inverted block letters used as stimuli in Experiments 1 and $2 \ldots \ldots . .20$

Figure 3. Experiment 1. Mean reaction times and error rates as a function of Display Type and Change Size with $95 \%$ confidence intervals.

Figure 4. Experiment 1. Mean reaction times and error rates as a function of Orientation and Change Size for each Display Type with $95 \%$ confidence intervals.

Confidence intervals were computed using the MSEs from the 2 (Orientation:

Upright vs. Inverted) by 3 (Change Size: 1 vs. 3 vs. 4 features) ANOVAs for each level of Display Type

Figure 5. Experiment 2. Mean reaction times and error rates as a function of Display

Type and Change Size with $95 \%$ confidence intervals.

Figure 6. Experiment 2. Mean reaction times and error rates as a function of Display

Type and Orientation with $95 \%$ confidence intervals.

Figure 7. Experiment 2. Mean reaction times and error rates as a function of Orientation and Change Size for each level of Display Type with $95 \%$ confidence intervals. ....35 


\section{Introduction}

The ability to perceive, retain and compare visual information across time is fundamental to our ability to accurately monitor the world around us. As such, change detection has been a matter of keen theoretical interest in cognitive psychology, with researchers attempting to better understand how people are able to compare and contrast different sources of information. Change detection is also widely studied in applied cognitive research, as it provides a means of assessing the ability to accurately monitor visual environments, whether it is in driving, flying, or any other type of task that requires consistent updating of visual information to maintain good Situation Awareness.

Change detection experiments often involve displaying two images separated by a blank interstimulus interval (ISI) at the same location. Notably, the flicker paradigm consists of continuously alternating an original $(\mathrm{A})$ and modified $\left(\mathrm{A}^{1}\right)$ image, with a blank ISI in between them (Rensink, O'Regan \& Clark, 1997). The images alternate until an observer detects the change between $\mathrm{A}$ and $\mathrm{A}^{1}$. However, while the flicker paradigm uses images that appear in the same spatial location to gauge the ability to detect change, this may not accurately represent how changes are detected in real-world settings given that the objects we monitor in our environments are often moving. An object must be mentally tracked such that it can be recognized as the same object even though it may later appear in a different spatial location. This apparent difference between how change detection is commonly operationalized in the laboratory and how it occurs in real-world conditions has prompted the following question: How does the process of detecting changes differ when temporally separated objects are compared across different locations rather than at the same location? 


\section{Spatial Location and Iconic Memory}

Research has shown that the physical separation between two sequentially presented displays affects whether they can be compared based on information stored in iconic memory (Irwin, 1991; 1992; Phillips, 1974). Iconic memory quickly and accurately stores large amounts of visual information for a brief period of time (see Figure 1 for a diagram of iconic memory and short-term memory). Therefore, comparisons of temporally separated images are more efficient if the Inter-Stimulus Interval (ISI: the time between the offset of the first image and the onset of the second image) is within the duration of iconic memory. However, this is only true if the images overlap completely (i.e., are not spatially separated). In Phillips' research, a dot-pattern (A) was presented for $33 \mathrm{~ms}$, followed by an ISI that varied between 20 and $600 \mathrm{~ms}$, which was then followed by a changed dot pattern $\left(\mathrm{A}^{1}\right)$, which differed from $\mathrm{A}$ by the displacement of a single dot. Half of the trials were overlapping, where $\mathrm{A}^{1}$ appeared in the same location as $\mathrm{A}$ and the other half shifted, where $\mathrm{A}^{1}$ was shifted a distance of one cell-width from the location of $\mathrm{A}$. The size of the displays was also varied by presenting dots in random cells of either a $5 \times 5$ matrix or an $8 \times 8$ matrix. Phillips reported significantly faster RTs and lower error rates in the overlapping condition than in the shifting condition, but only if the ISI was less than $300 \mathrm{~ms}$. At ISIs higher than $300 \mathrm{~ms}$, Phillips reported no difference in RTs and error rates between overlapping and shifting displays. Similarly, the size of the dot matrices was reported to have no affect on RTs or error rates at the lowest ISI $(20 \mathrm{~ms})$ in the overlapping condition, but the size of dot matrices significantly affected RTs and error rates at the $20 \mathrm{~ms}$ ISI in the shifting condition. Phillips interpreted this interaction between display size and moving versus overlapping displays as evidence that iconic memory is tied to location, whereas visual STM is not (see also Irwin, $1991 ; 1992)$. 


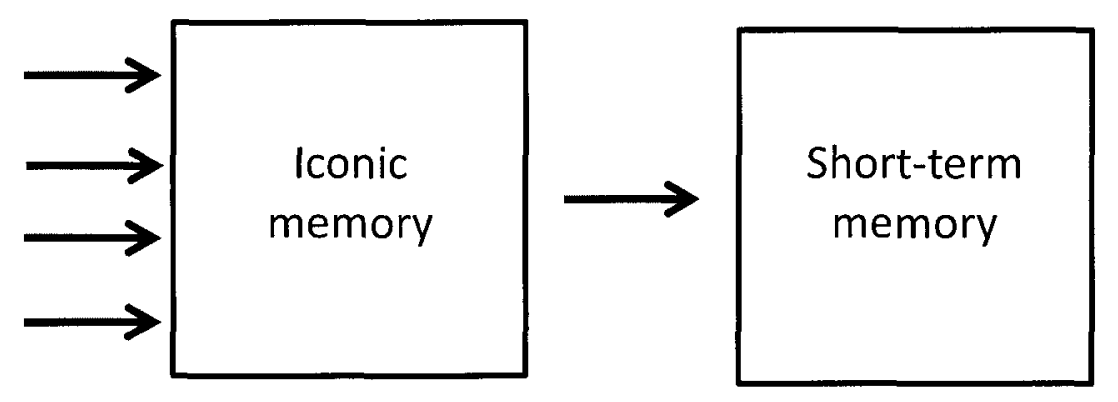

Figure 1. Diagram of iconic memory and short-term memory.

The efficiency of using iconic memory to compare temporally separated objects that appear in the same location was also observed by Shore and Klein (2000), who reported that changes were detected more quickly when an original $(\mathrm{A})$ and modified $\left(\mathrm{A}^{1}\right)$ image alternated in the same location compared to when $\mathrm{A}$ and $\mathrm{A}^{1}$ were (simultaneously) displayed side-by-side. Shore and Klein asked participants to detect the change in an image of a naturalistic scene in either a flicker or a simultaneous condition. In the flicker condition, $\mathrm{A}$ and $\mathrm{A}^{1}$ alternated in the same location on a computer screen until the change was localized. Display durations of A and $\mathrm{A}^{1}$ were $555 \mathrm{~ms}$, with an ISI that was within the duration of iconic memory $(120 \mathrm{~ms})$. In the simultaneous condition, $\mathrm{A}$ and $\mathrm{A}^{1}$ were photographs placed side-by-side. Participants compared the adjacent photographs until they identified the difference. Shore and Klein reported that RTs in the simultaneous condition were, on average, twice as long as those in the flicker condition. This is consistent with Phillips' (1974) finding that spatial overlap facilitates change detection when the temporal separation between $\mathrm{A}$ and $\mathrm{A}^{1}$ is within the duration of iconic memory. 
Shore and Klein (2000) also provided evidence that participants' visual representations of $\mathrm{A}$ and $\mathrm{A}^{1}$ were different in the simultaneous and flicker conditions. Previous work by Rensink, O'Regan and Clark (1997) had shown that participants localize changes more quickly if they are of central interest than if they are of marginal interest to the scene's context. For example, a person's face featured prominently in a scene may be of central interest, whereas a tree in the background may be of marginal interest. Shore and Klein used the same images and changes used by Rensink et al., including the central interest and marginal interest classifications. However, on half of Shore and Klein's trials, the images appeared upside-down. Shore and Klein suggested that if the faster RTs for central interest changes was due to their relevance within the context of images, then inverting the images should eliminate (or reduce) the central versus marginal interest effect, because presenting images upside-down should interfere with participants' interpretation of the images' context. Shore and Klein reported that displaying the images upside-down reduced the central versus marginal interest effect in the simultaneous condition, but not in the flicker condition. That is, in the simultaneous condition, central interest changes were only detected more quickly than marginal interest changes when images were upright. In contrast, displaying images upside-down had no effect on the central versus marginal interest effect in the flicker condition; central interest changes were detected more quickly than marginal interest changes regardless of whether the images were upright or upside-down. Shore and Klein argued that this three-way interaction between display type (flicker or simultaneous), image orientation (upright or upside-down), and change type (central or marginal interest), is evidence that central interest changes are not always detected more quickly than marginal interest changes because of their relevance to the image's context. If the central versus marginal interest effect was always due to contextual cues, then inverting the images in Shore and Klein 
should have disrupted the central versus marginal interest effect equally in both the flicker and simultaneous conditions. Shore and Klein explained this inconsistency by suggesting that the central versus marginal effect may have been produced by different sources, depending on whether the change occurred in the flicker or simultaneous paradigm. Shore and Klein noted that there are at least two explanations for why a changing object will be designated as central or marginal. First, central interest objects could have had more relevance to the image's context. Second, central interest objects could have had more perceptual salience than the other objects. Therefore, it is possible central interest changes were detected more quickly than marginal interest changes for two different reasons: (1) because participants relied on the context of images to guide their search or (2) the lower-level perceptual salience of objects captured their attention. Shore and Klein suggested that inverting images likely reduced the central versus marginal interest effect in the simultaneous paradigm because participants were searching for changes based on the higher-level context of images. Conversely, in the flicker paradigm, participants may have searched for changes based on lower-level cues (e.g., salience) which would likely not be affected by inverting the images.

Shore and Klein's (2000) finding that changes are detected more quickly in the flicker condition than in the simultaneous condition when the ISI between the two changing images is short (i.e., $120 \mathrm{~ms}$ ) is consistent with previous findings that iconic memory facilitates the comparison of overlapping objects, but not spatially separated ones (Phillips, 1974; Irwin, 1991). Although Shore and Klein's claim that changes were detected using contextual cues in the simultaneous condition and object salience in the flicker condition is consistent with their data, it remains to be seen whether this was due to the lack of spatial overlap in the simultaneous condition or to the additional time required to make comparisons in the simultaneous condition. 
The $120 \mathrm{~ms}$ ISI in the flicker condition allows participants to detect changes based on featural information about $\mathrm{A}$ and $\mathrm{A}^{1}$ stored in iconic memory. Therefore, participants may have focused more on featural aspects in the flicker paradigm, because they were able to make comparisons based on low-level information stored in iconic memory. In lieu of relying on bottom-up information stored in iconic memory in the simultaneous condition, participants may have used contextual information provided by top-down processing to help guide their search through images stored in short-term memory. However, it is also possible that participants focussed on image context in the simultaneous condition because more time was available to encode an image's contents to a deeper level in the simultaneous condition given that there was no limit to how long image $\mathrm{A}$ could be processed before comparing it to image $\mathrm{A}^{1}$.

Research by Richards (2002) showed that the likelihood of processing an image/object at a higher level (i.e., using identity or semantic category information) increases with exposure duration. In Richards' study participants searched for changing objects in alternating arrays of letters or unfamiliar objects. The effect that familiarity had on performance was found to increase as SOA increased. Therefore, contextual information may have been available to participants in Shore and Klein's simultaneous condition because more time was available to process the images - not because the images were spatially separated. Whether or not a spatial manipulation in and of itself affects the relative contributions of lower and higher level processing in detecting changes remains to be seen. This will be addressed in the present thesis by holding timing parameters constant across conditions where $A$ and $A^{1}$ either appear in the same location or in different locations.

In sum, the results of Phillips (1974) and Shore and Klein (2000) show that detecting changes in temporally separated objects is easier when they appear in the same location than 
when they appear in different locations, but only if the ISI separating the two objects is within the duration of iconic memory. However, it is unclear whether comparing spatially separated objects based on information stored in STM forces the observer to rely on top-down information about those objects, or if, when restricted to short ISIs, observers will continue to use the same featural information that allows changes to be detected in iconic memory.

\section{Spatial Location and Short-Term Memory}

Some research on how spatial location affects memory-based comparisons of temporally separated objects indicates that spatial location does not play a prominent role when the information being compared is stored in short-term memory (Phillips, 1974; Irwin, 1991; 1992). However, other studies provide evidence that it is more difficult to compare objects that appear in different spatial locations than when they appear in the same location, even when the ISI between object presentations is are beyond the duration of iconic memory (Brockmole \& Irwin, 2005; Williams \& Simons, 2000). Furthermore, Brockmole and Irwin, and Williams and Simons provided evidence that it may be more difficult to shift a detailed visual representation of an object to a new spatial location than to hold it in the same location. This may encourage the recruitment of different processing strategies for comparing spatially separated or overlapping objects when the ISI is beyond the duration of iconic memory.

Williams and Simons (2000) reported faster responses to changes in objects that overlapped than in objects that appeared in different locations when the ISIs were well beyond the duration of iconic memory. Stimuli were single objects that scrolled across a computer screen or alternated in the same spatial location. In the scrolling condition, an object (A) scrolled across the horizontal axis of a computer screen and was occluded by a black square in the center of the screen. The object then emerged on the other side of the square as either the same (A) or a 
changed object $\left(\mathrm{A}^{1}\right)$. The participants' task was to indicate whether the object had changed when it emerged from behind the occlusion. The objects were collections of geons arranged to form single objects. The number of parts changing was manipulated by replacing one, two or three of the object's geons. In the overlap condition, $A$ and $\mathrm{A}^{1}$ appeared in the same spatial location. Object A was displayed for $1660 \mathrm{~ms}$, followed by a $1660 \mathrm{~ms}$ blank ISI, followed by a 1660 presentation of $\mathrm{A}$ or $\mathrm{A}^{1}$. Williams and Simons reported that participants responded approximately $650 \mathrm{~ms}$ faster in the overlap condition than in the scrolling condition, while overall accuracy did not change. Furthermore, a regression analysis revealed that accuracy in the overlap condition was best predicted by the size of the change (number of pixels changing), whereas accuracy in the scrolling condition was best predicted by the number of parts that changed. Williams and Simons speculated that this difference was caused by the scrolling condition encouraging a structural/verbal encoding style sensitive to the composition of geons, whereas differences in the overlap condition were more likely to be detected based on physical characteristics (i.e., the number of pixels that changed).

Williams and Simons' (2000) finding that changes are more difficult to detect when A and $\mathrm{A}^{1}$ appear in different locations than in the same location is inconsistent with the previous finding that visual STM representations are not linked to spatial location (Phillips, 1974; Irwin, 1991; 1992). An explanation for the RT difference between Williams and Simons' overlap and scrolling conditions may come from their finding that changes to overlapping objects were best detected when the change was physically large, but changes to moving objects were best detected when the number of changing parts was large. It is possible that it is more difficult to maintain a veridical representation of $\mathrm{A}$ in order to compare with $\mathrm{A}^{1}$ if $\mathrm{A}$ and $\mathrm{A}^{1}$ appear in different locations than in the same location. Thus, participants in Williams and Simons may 
have employed a different encoding strategy when comparing $\mathrm{A}$ and $\mathrm{A}^{1}$ in the scrolling condition than in the overlap condition, which was less sensitive to the number of pixels that changed.

Support for the claim that it is more difficult to shift a veridical representation of visual information from one location to another than it is to hold it in the same location comes from Brockmole and Irwin (2005), who reported that it was more difficult to shift a mental representation of a dot pattern stored in visual STM to a new spatial location than to hold the pattern in the same spatial location. Cells of two grids ( $A$ and $\left.A^{1}\right)$ were randomly populated with dots such that only one cell of both grids did not contain a dot. Participants were to identify the cell that was not occupied by a dot. Within a trial, the grid either appeared in the same spatial location or in different locations. In the spatial shift condition, $A$ and $A^{1}$ were separated by twenty degrees in horizontal visual angle whereas $\mathrm{A}$ and $\mathrm{A}^{1}$ occupied the same space in the overlap condition. Brockmole and Irwin reported that accuracy did not differ across the two conditions. However, in the spatial shift condition, participants foveated on the cells of $\mathrm{A}^{1}$ that were occupied by dots in A. Conversely, in the spatial overlap condition, participants made very few eye movements; participants moved their eyes as little as possible while waiting for $\mathrm{A}^{1}$ to appear. Brockmole and Irwin suggested that, in the shift condition, participants may have deployed overt attention to grid locations in $\mathrm{A}^{1}$ that were previously occupied by dots in $\mathrm{A}$ in order to rehearse pattern A in memory. This implies that veridical visual representations may be difficult to maintain across different spatial locations. Therefore, it is possible that Williams and Simons' (2000) overlap condition allowed A and $\mathrm{A}^{1}$ to be compared based on their veridical representations, whereas further encoding was required to make comparisons in the scrolling condition because veridical representations cannot be shifted across spatial locations. 
In sum, Williams and Simons' (2000) results indicate that temporally separated objects that appear in different locations are more difficult to compare than temporally separated objects that appear in the same location. Williams and Simons, and Brockmole and Irwin (2005) also provided evidence that the visual information used to compare two temporally separated objects may change depending on whether the objects are spatially separated or overlap. The effect of spatial location on change detection reported by Williams and Simons, and Brockmole and Irwin stands in contrast to prior suppositions that spatial location plays no role in comparisons made based on information stored in STM (Phillips, 1974; Irwin, 1991; 1992). Therefore, further investigation into whether or not spatial location affects the comparison of temporally separated displays in STM is warranted.

\section{Research Questions}

The research to date on the effect of spatial location on change detection shows that it is often easier to detect changes in objects that appear in the same location than objects that appear in different locations (Brockmole \& Irwin, 2005; Phillips, 1974; Shore \& Klein, 2000; Williams \& Simons, 2000). However, the following questions as to the qualitative differences between detecting changes in overlapping versus shifting objects remain open:

(1) If timing parameters are held constant, are observers able to use top-down information about objects to compensate for a relative lack of bottom-up information when comparing non-overlapping objects versus overlapping objects when the ISI is within the duration of iconic memory?

(2) With temporal separations beyond the duration of iconic memory, is it more difficult to shift a one-to-one visual representation of objects stored in STM to a new location to a new location than to hold it in the same location? And if so, is this difficulty reflected in a 
greater reliance on top-down information for STM-based comparisons of shifting versus overlapping objects when timing parameters are held constant?

Both questions (1) and (2) can be addressed with a change detection paradigm that includes the following manipulations. First, a spatial manipulation where $\mathrm{A}$ and $\mathrm{A}^{1}$ either appear in the same location or different locations would allow the effect of spatial location to be assessed. Second, a bottom-up manipulation that affects the visual characteristics of changes would provide an indication of the extent to which comparisons are being made with bottom-up visual information. The size of a bottom-up manipulation's effect should increase or decrease as a function of whether observers focus more heavily on bottom-up or top-down information about changing objects, respectively. Third, a semantic manipulation that affects observers' ability to recruit top-down information about changing objects would provide an indication as to the extent to which participants rely on top-down information to make comparisons. The size of a semantic manipulation's effect should increase or decrease as a function of whether observers rely more heavily on top-down or bottom-up sources of information about changing objects, respectively.

\section{Conceptualizing Top-Down \& Bottom-Up Processing}

Work by Richards (2002) and Tovey (2011) has provided a framework for uncovering the relative contributions of bottom-up and top-down sources of information for the detection of a single change. Richards showed that, when stimulus onset asynchronies (SOA) are short, change detection performance is strongly affected by the number of features that change between $\mathrm{A}$ and $\mathrm{A}^{1}$ and therefore concluded that bottom-up processing is used to detect changes under these conditions. Richards further showed that as SOA increases and participants have more time to consolidate representations of objects in STM, change detection becomes more strongly affected by top-down information (i.e., object familiarity). Tovey extended this finding by 
manipulating the number of features changing as in Richards as well as the orientation of letters (upright vs. inverted) within a single within subjects experiment and showed that the impact of change size on the ability to detect changes is modulated by whether the stimulus is familiar (an upright letter) or not (an inverted letter). That is, bottom-up information (number of feature changes) has a larger influence on change detection when the changing letter was unfamiliar (inverted) than when it was familiar (upright).

Richards' (2002) analysis was based on the hypothesis that the amount of time provided to encode $\mathrm{A}$ and $\mathrm{A}^{1}$ affects whether changes are detected based on either the featural properties or the higher level attributes (e.g., name, category, etc.) of the changing stimuli. Richards further suggested that giving participants more time to encode the displays should promote the consolidation of the visual information into more robust object representations that can be used to efficiently detect changes. Thus, there is a shift from feature-based processing to object-based processing as the time provided to encode the displays increases. Richards did not suggest that feature-based comparisons should be confined to comparisons made with information stored in iconic memory, but rather featural information is likely stored in STM before enough time has passed for it to be consolidated into an object-level representation.

According to Richards (2002), feature-based processing relies on information stored early in the course of visual processing (e.g., iconic memory). As such, feature-based processing is limited to basic bottom-up information and is devoid of semantics. Object-based processing occurs later in the course of visual processing and relies on the activation of STM representations created by the consolidation of features into distinct objects. Richards proposed the following characteristics and functional differences between feature and object-based processing. First, object-based representations take time to consolidate, as evidenced by the finding that it takes 
approximately $50 \mathrm{~ms}$ to consolidate a single object into STM (Vogel, Woodman \& Luck, 2006). Second, object-based representations can be stored more efficiently in memory than their component features. Research shows that the capacity requirements of encoding a single feature in visual STM are the same as encoding an entire object (Luck \& Vogel, 1997). Third, objectbased representations allow for the comparison of items across multiple visual and non-visual dimensions. For example, letters stored in STM may be compared in terms of their featural characteristics, verbal code or categorical information. Richards therefore claimed that the presence of a change size effect (i.e., change detection performance increases as the number of changing features increases) indicates that feature-based processing was used to detect change. If, on the other hand, the object as a whole - and not its component features - are being used to detect a change (i.e., object-based processing), then the number of individual features changing within that object should not affect change detection performance. Richards further argued that object-based processing is more likely to occur when more time is provided (by extending the durations of $\mathrm{A}$ and $\mathrm{A}^{1}$ or the ISI) to consolidate featural information into object-based representations in STM.

Richards (2002) used a flicker paradigm where participants searched for a target amongst distractors displayed at random locations on an invisible $6 \times 6$ matrix. The target was a single changing letter or number, and distractors were unchanging letters or numbers. Richards varied the number of features changing in the target (change size) and the number of distractors (set size). Critically, the display durations of $\mathrm{A}$ and $\mathrm{A}^{1}$ and the ISIs were varied between subjects. Display durations were 200 or 370 ms. ISI lengths were $80,250,500$ or 1000 ms. Richards reported that the change size effect decreased with each increase in either display duration or ISI length, to the extent that the change size effect was not significant at an ISI of $1000 \mathrm{~ms}$. Search 
efficiency also increased with display and ISI durations, as participants required fewer alternations of $\mathrm{A}$ and $\mathrm{A}^{1}$ to successfully locate the change. The consolidation of object-based representations did not seem to be strongly tied to whether it was the display duration or ISI that increased. Rather, Richards reported that the strongest determinant of object-based processing was simply the amount of time between the onsets of $\mathrm{A}$ and $\mathrm{A}^{1}$ (i.e., display duration + ISI).

To further support the claim that improved change detection performance at longer ISIs was attributable to a shift from feature-based to object-based processing, Richards (2002) argued that change detection should be more difficult with unfamiliar items than with familiar items, as familiar items are more likely to be processed as objects than unfamiliar items. Long-term memory representations of familiar objects are likely to facilitate the consolidation and maintenance of familiar items in STM and allow participants to make comparisons based on nonvisual attributes (e.g., verbal labels, categorical information). Conversely, unfamiliar items do not have these long-term memory representations and must therefore be compared using bottomup information. To test this hypothesis, Richards compared change detection performance between unfamiliar objects (i.e., a circle with several lines extending from the centre to the radius - a wheel with spokes) and familiar objects (i.e., letters and numbers). Change size for the unfamiliar items was manipulated by removing or adding spokes that were identical in size to the features that changed in the letters and numbers. The results were consistent with Richards' predictions: the change size effect was larger for unfamiliar stimuli than for familiar stimuli. Moreover, the change size effect for unfamiliar stimuli was significant at longer display durations and ISIs, whereas it was eliminated at these longer durations for the familiar stimuli.

One problem with Richards' (2002) methodology is that the unfamiliar stimuli were drastically different than the familiar stimuli in terms of their physical appearance. Thus, it is 
possible that Richards' results were due to these physical differences rather than to familiarity. Tovey (2011) addressed this issue by using inverted letters instead of Richards' "wheels" as unfamiliar stimuli, thus holding the physical characteristics of the stimuli constant across familiarity conditions. As in Richards, Tovey manipulated change size and set size in a flicker paradigm. Stimulus arrays consisted of a target amongst distractors displayed in randomly selected locations on an invisible $6 \times 6$ matrix. Block letters were used as stimuli. Critically, the orientation of letters was manipulated such that every letter in the display was reflected over its $\mathrm{x}$-axis on half of the trials to create unfamiliar stimuli. Display durations were $200 \mathrm{~ms}$ and ISIs were $80 \mathrm{~ms}$. Changes to familiar (upright) letters were detected more quickly than changes to unfamiliar (inverted) letters. Furthermore, familiarity interacted with change size, in that the change size effect was larger for unfamiliar stimuli than for familiar stimuli, suggesting that the familiarity of upright letters facilitated their consolidation into object-based representations. On the other hand, the unfamiliarity of inverted letters prevented or interfered with them being consolidated into object-based representations in STM, therefore forcing the system to rely on bottom-up (feature-based) processing to detect the change, hence the larger change size effect for unfamiliar letters. Thus, by jointly manipulating change size and familiarity within a single within-subjects design, Tovey successfully demonstrated the combined effects of bottom-up and top-down processing on change detection.

One thing that is not clear from Richards' (2002) and Tovey's (2011) findings is the extent to which decreases in the effect of change size may be due to comparing $A$ and $\mathrm{A}^{1}$ on nonvisual dimensions. While it is plausible that participants may compare items based on verbal codes or categorical information, it is equally plausible that object-based representations would allow for more efficient comparison of $\mathrm{A}$ and $\mathrm{A}^{1}$ in terms of their visual characteristics. Stolz and 
Jolicoeur (2004) showed that smaller changes are more likely to be missed than large changes, using the same LED characters and change size manipulation used by Richards (2002). Stolz and Jolicoeur used a one cycle paradigm (instead of a flicker paradigm) where display A was shown for $200 \mathrm{~ms}$, followed by an $84 \mathrm{~ms}$ ISI, followed by $\mathrm{A}^{1}$, which remained on the screen until response. Error rates increased significantly as change size decreased $(9.2 \%, 11.3 \%$, and $13.4 \%$ for four, three and two-feature changes, respectively), which shows that smaller changes are more likely to be missed than larger changes. If a change is missed in the flicker paradigm (e.g., Richards, 2002; Tovey, 2011), then the entire display must be re-searched until the changing target is detected. The process of re-searching a display for a changing target is more likely to occur for smaller than for larger changes (because smaller changes are more likely to be missed), thus increasing RTs more for smaller changes than for larger changes. Therefore, although comparing $\mathrm{A}$ and $\mathrm{A}^{1}$ on non-visual dimensions could result in a smaller effect of change size in Richards' paradigm, it is equally plausible that the change size effect would be smaller as participants consolidate $A$ and $A^{1}$ into more robust visual representations such that they are less likely to miss the change (see also Mitroff, Simons \& Franconeri, 2002). Familiarity may also increase the efficiency of the comparisons between $\mathrm{A}$ and $\mathrm{A}^{\mathbf{1}}$, as corresponding representations of familiar items in long-term memory should facilitate the retention of each item's identifying visual features in STM. Therefore, neither Richards' nor Tovey's paradigm allows one to determine whether a smaller effect of change size results from items being compared on nonfeatural dimensions or simply from consolidating more robust object-based representations.

Manipulating the spatial location of $\mathrm{A}$ and $\mathrm{A}^{1}$ may provide clarification as to whether a reduction in the change size effect may occur due to participants comparing letters based on their visual characteristics or based on non-visual dimensions, such as verbal codes or categories. If 
display durations and ISIs are held constant across two conditions where $\mathrm{A}$ and $\mathrm{A}^{1}$ appear in the same or different locations, then there is no reason to expect that better-consolidated objectbased representations would be more likely in one condition than the other given Richards' (2002) assertion that the shift from feature-based to object-based processing is best predicted by the time between the onset of the two displays (i.e., display duration + ISI). The robustness of the representations being compared should not depend on whether $\mathrm{A}$ and $\mathrm{A}^{1}$ appear in the same or different locations. However, if it is more difficult to compare objects on visual dimensions when $A$ and $\mathrm{A}^{1}$ appear in different locations than in the same location, then non-visual information about objects may be recruited for comparison under these conditions. Such an effect would result in a smaller effect of change size when $A$ and $A^{1}$ appear in different locations than when they appear in the same location, even if display duration and ISI are held constant. One would also expect to observe a larger familiarity effect when the objects appear in different locations, as comparisons are more likely to be made using higher-level (i.e., non-featural) dimensions. In sum, Richards (2002) and Tovey (2011) provide a framework for examining the relative contributions of bottom-up and top-down information to change detection in a flicker paradigm. Richards showed that the change size effect decreases as the time provided to consolidate visual information into representations (in STM) increases. Richards also showed that familiarity facilitates the detection of changes, especially at longer display durations and ISIs that allow for the activation of an object's representation in STM. Tovey showed that by inverting familiar stimuli (e.g., letters) one can manipulate familiarity without altering the physical characteristics of the stimuli. This allowed for the concurrent manipulation of a factor that affects bottom-up processing (i.e., change size) and a factor that affects top-down processing 
(familiarity). Tovey reported that change size and familiarity interact such that the change size effect is smaller for familiar objects than for unfamiliar ones.

\section{Present Research}

This thesis used the framework set by Richards (2002) and Tovey (2011) to explore the combined effects of spatial location (overlapping vs. shifting), feature change size (small vs. large), and familiarity (familiar vs. unfamiliar) on change detection at short (80 ms) and long (500 ms) ISIs. ISI was manipulated with the intent of enabling bottom-up (feature-based) processing at a short ISI $(80 \mathrm{~ms})$ and promoting top-down (semantic) processing at a long ISI (500 ms) to determine whether spatial location differentially affects the extent to which participants rely on bottom-up and top-down processing when comparing objects to detect a change.

This thesis extends the research on the effects of spatial location in change detection by comparing change detection across conditions where $\mathrm{A}$ and $\mathrm{A}^{1}$ appear in the same location (Overlap condition) and where they appear in different locations (Shift condition), while holding timing parameters constant. Factors that affect low-level processing (Change Size) and highlevel processing (Familiarity) were manipulated in order to assess the relative contributions of low-level and high-level information to detecting across the Overlap and Shift conditions. Experiment 1 explored the effects of spatial location on change detection when the ISI separating $\mathrm{A}$ and $\mathrm{A}^{1}$ was within the duration of iconic memory $(80 \mathrm{~ms})$. If higher level information is relied on more heavily when $\mathrm{A}$ and $\mathrm{A}^{1}$ shift than when they overlap, then there should be a larger effect of the semantic manipulation (i.e., Familiarity) in the Shift condition than in the Overlap condition. Similarly, if low-level featural information is relied on less when $\mathrm{A}$ and $\mathrm{A}^{1}$ shift than 
when they overlap, then the effect of the change size manipulation should be smaller in the Shift condition than in the Overlap condition. In Experiment 2, the ISI was increased to $500 \mathrm{~ms}$ in order to examine the effects of spatial location on change detection when STM is used to make comparisons. If it is more difficult to shift a STM-based visual representation to a new spatial location than to hold it in the same location (see Brockmole \& Irwin, 2005; Williams \& Simons, 2000), then top-down information may play a larger role in the Shift condition than the Overlap condition, as it will help participants to retain the structures of upright letters across a spatial shift. A greater reliance on top-down information in the Shift condition will be manifested as a larger effect of letter orientation in the Shift condition than in the Overlap condition.

Experimental Paradigm. Three variables were manipulated. First, spatial location was manipulated by having displays either appear in the same location (Overlap condition) or in different locations (Shift condition). Second, the number of features that changed ( 1 vs. 3 vs. 4 ) was manipulated under the assumption that is would affect a comparison process that relied primarily on bottom-up information. Third, the familiarity of the changing object (upright familiar vs. inverted - unfamiliar) was manipulated to measure the effects of top-down processing in change detection. Inverting stimuli will impair their recognisability, but this impairment cannot be attributed to bottom-up changes, given that the featural composition of an upright and inverted stimulus is identical. As such, any effect that inverting the stimulus has on the comparison process is best explained in terms of its influence on higher-level processing.

\section{Experiment 1}

The objective of Experiment 1 was to examine the effect that a spatial manipulation (i.e., overlapping vs. shifting displays) has on people's ability to detect changes when relatively little time is available to encode the original display $(\mathrm{A})$ and the changed display $\left(\mathrm{A}^{1}\right)$. Experiment 1 
compared change detection performance in a standard flicker paradigm where alternating displays ( $\mathrm{A}$ and $\mathrm{A}^{1}$ ) appeared at the same location (Overlap condition) or when the displays shifted approximately 1 degree in horizontal visual angle (Shift condition). The display durations of $A$ and $A^{1}(200 \mathrm{~ms})$ and the duration of the ISIs $(80 \mathrm{~ms})$ were held constant across the Overlap and Shift conditions. A and $\mathrm{A}^{1}$ consisted of arrays of block letters (see Figure 2) displayed at random locations in the cells of an invisible $6 \times 6$ matrix. The target was a single changing letter amongst non-changing (distractor) letters. The number of features changing (Change Size) and the orientation of stimuli (Orientation) were manipulated.

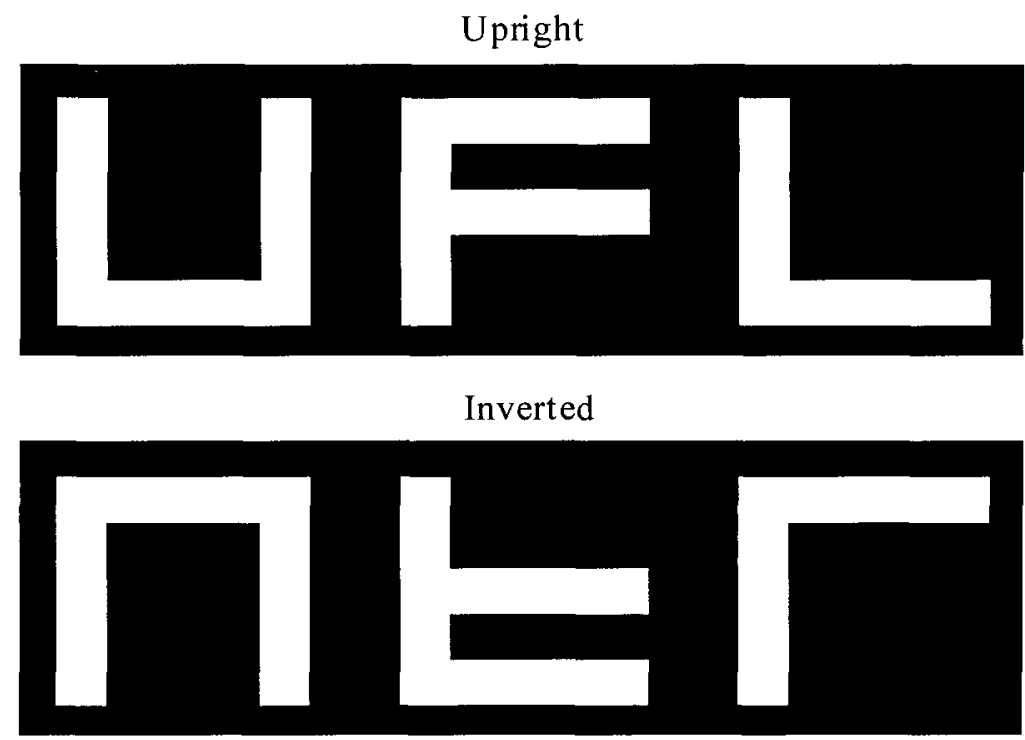

Figure 2. Upright and inverted block letters.

\section{Predictions}

It was expected that performance would be substantially worse in the Shift condition than in the Overlap condition, based on previous experiments reporting longer RTs and/or higher error rates with non-overlapping than with overlapping stimuli at ISI durations similar to that used in this experiment (Phillips, 1974; Irwin, 1991). The unavailability of quickly-stored 
bottom-up information in iconic memory in the Shift condition could have influenced the effects of the Change Size and Orientation manipulations in two ways. If participants could not use topdown information about $\mathrm{A}$ and $\mathrm{A}^{1}$ in the Shift condition to compensate for the unavailability of bottom-up information stored in iconic memory, then the effect of Orientation should have been equal in the Overlap and Shift conditions. Similarly, if participants could not have used top-down information about $\mathrm{A}$ and $\mathrm{A}^{1}$ in the Shift condition to compensate for the unavailability of bottomup information in stored in iconic memory, then the effect of Change Size should have been larger in the Shift condition than the Overlap condition, because participants would have been able to compare A and $\mathrm{A}^{1}$ based solely on bottom-up information stored in STM in the Shift condition, whereas they would have been able to compare $\mathrm{A}$ and $\mathrm{A}^{1}$ based on bottom-up information stored in iconic memory and STM in the Overlap condition. Conversely, if top-down information could be used to compensate for the lack of bottom-up information about $\mathrm{A}$ and $\mathrm{A}^{1}$ in the Shift condition, then the effect of Orientation should have been greater in the Shift condition than the Overlap condition. It was not clear whether and to what extent Change Size would differ in the Shift condition relative to the Overlap condition, if participants could use topdown information to compensate for the lack of bottom-up information in the Shift condition, because it was not clear to what extent this top-down information may facilitate comparisons in the Shift condition. If top-down information facilitates retention of the visual aspects of $A$ and $A^{1}$ in STM, then the effect of Change Size may have been equal in the Overlap and Shift conditions. Conversely, if top-down information about $\mathrm{A}$ and $\mathrm{A}^{1}$ allows for comparisons of letters on nonvisual dimensions, then the effect of Change Size should have been smaller in the Shift than the Overlap condition.

\section{Method}


Participants: Thirty-five Carleton University undergraduate students participated for $1 \%$ course credit.

Design: The experiment was a 2 (Display Type: Overlap vs. Shift) x 3 (Change Size: 1, 3 or 4 features) x 2 (Orientation: Upright vs. Inverted), within-subjects design. The Overlap and Shift conditions were blocked, and Change Size and Orientation were mixed within each block, such that there were an equal number of trials for each level of those factors. Display Type was counterbalanced across participants such that half the participants received the Overlap condition followed by the Shift condition, and the other half received the reverse order. The experiment consisted of 288 trials, and required approximately 45 minutes to complete.

Stimuli and Apparatus: The experiment was programmed in Inquisit, by Millisecond Software (2010) and implemented on an Intel-based computer running Windows Vista. Stimuli were presented on a 24" Dell Viewsonic LCD monitor at a resolution of $1920 \times 1200$ with a refresh rate of $60 \mathrm{~Hz}$. Responses were collected using a USB keyboard and a USB mouse. Stimuli were arrays of white block letters. Each letter was $100 \times 100$ pixels in size. The block letters covered an area of approximately $2.7 \mathrm{~cm} \times 2.7 \mathrm{~cm}$ on the computer screen. The original array (A) consisted of 10 letters. The modified array $\left(\mathrm{A}^{1}\right)$ was identical to $A$, except that one letter (the target) changed to a different letter. The letters were F, L and U. The target was either an $\mathrm{L}$ alternating with a $\mathrm{U}$ (1 feature change), an $\mathrm{L}$ alternating with an $\mathrm{F}$ ( 3 feature change) or an F alternating with a $U$ ( 4 feature change). Targets and distractors were displayed in randomly selected cells of an imaginary $6 \times 6$ matrix. The matrix subtended a visual angle of roughly 18 degrees both vertically and horizontally. 
Procedure: All participants were tested individually in a quiet room. Task instructions were displayed on-screen. Participants completed four practice trials immediately before each of the two experimental blocks (Overlap and Shift).

The trial sequence was as follows: a fixation cross was displayed in the centre of the area that would be occupied by array A (in the center of the screen). The fixation cross remained on the screen for $500 \mathrm{~ms}$, at which time it was replaced by array A, which was presented for $200 \mathrm{~ms}$. After an $80 \mathrm{~ms}$ blank ISI, $\mathrm{A}^{1}$ was presented for $200 \mathrm{~ms}$, followed by another $80 \mathrm{~ms}$ blank ISI, A was then displayed again. This cycled until the participant responded, up to a maximum of 20000 ms.

Participants responded by pressing the spacebar to stop the RT clock, at which point the screen was blanked for $80 \mathrm{~ms}$. Array A was then displayed and participants used a mouse to indicate which stimulus was changing. Feedback was provided by displaying either the word "Correct" or "Error." The ITI was $1000 \mathrm{~ms}$.

\section{Results}

Response times: Response times for correct trials were submitted to Van Selst and Jolicoeur's (1994) recursive outlier removal procedure, which eliminated correct RTs that were 3 standard deviations above or below the mean RT for each condition for each participant. This procedure continued recursively until no further RTs beyond 3 standard deviations from the mean of each condition for each participant were found. This resulted in $2.24 \%$ of the trials being excluded. The remaining data were analyzed using a 2 (Display Type: Overlap vs. Shift) by 3 (Change Size: 1 vs. 3 vs. 4 features) by 2 (Orientation: Upright vs. Inverted) repeated measures ANOVA. 
There was a significant main effect of Display Type, $F(1,34)=119.5$, MSE $=570966, p$ $<.001$, partial $\eta 2=.778$, where participants detected changes more quickly in the Overlap condition (1568 ms) than in the Shift condition (2372 ms). The main effect of Change Size was significant $F(2,68)=95.7, \mathrm{MSE}=134172, p<.001$, partial $\eta^{2}=.738$, where RTs decreased as Change Size increased from 1-feature $(2303 \mathrm{~ms})$ to 3 -features $(1879 \mathrm{~ms})$ to 4 -features $(1724 \mathrm{~ms})$. The main effect of Orientation was also significant, $F(1,34)=4.8, \mathrm{MSE}=108221, p=.036$, partial $\eta^{2}=.123$. Participants detected changes in upright letters $(1933 \mathrm{~ms})$ more quickly than inverted letters (1999 ms).

The interaction between Display Type and Change Size was significant, $F(2,68)=18.7$, MSE $=88497, p<.001$, partial $\eta^{2}=.354$ (see Figure 3). The effect of Display Type was largest for 1 -feature changes, smaller for 3-feature changes and smallest for 4-feature changes. None of the other interactions were significant $(\mathrm{Fs}<1)$.

Post hoc analysis: A pairwise comparison with a Bonferroni adjustment was carried out on the effect of Orientation in the Overlap and Shift conditions. Changes in upright letters (1524 $\mathrm{ms}$ ) were detected more quickly than changes in inverted letters $(1612 \mathrm{~ms})$ in the Overlap condition, $M_{d t f f}=90.3, s_{\text {error }}=29.7, p=.005$, whereas changes in upright letters (2351 ms) were not detected significantly more quickly than changes in inverted letters $(2393 \mathrm{~ms})$ in the Shift condition, $M_{\text {deff }}=50.1, s_{\text {error }}=62.3, p=.427$. 


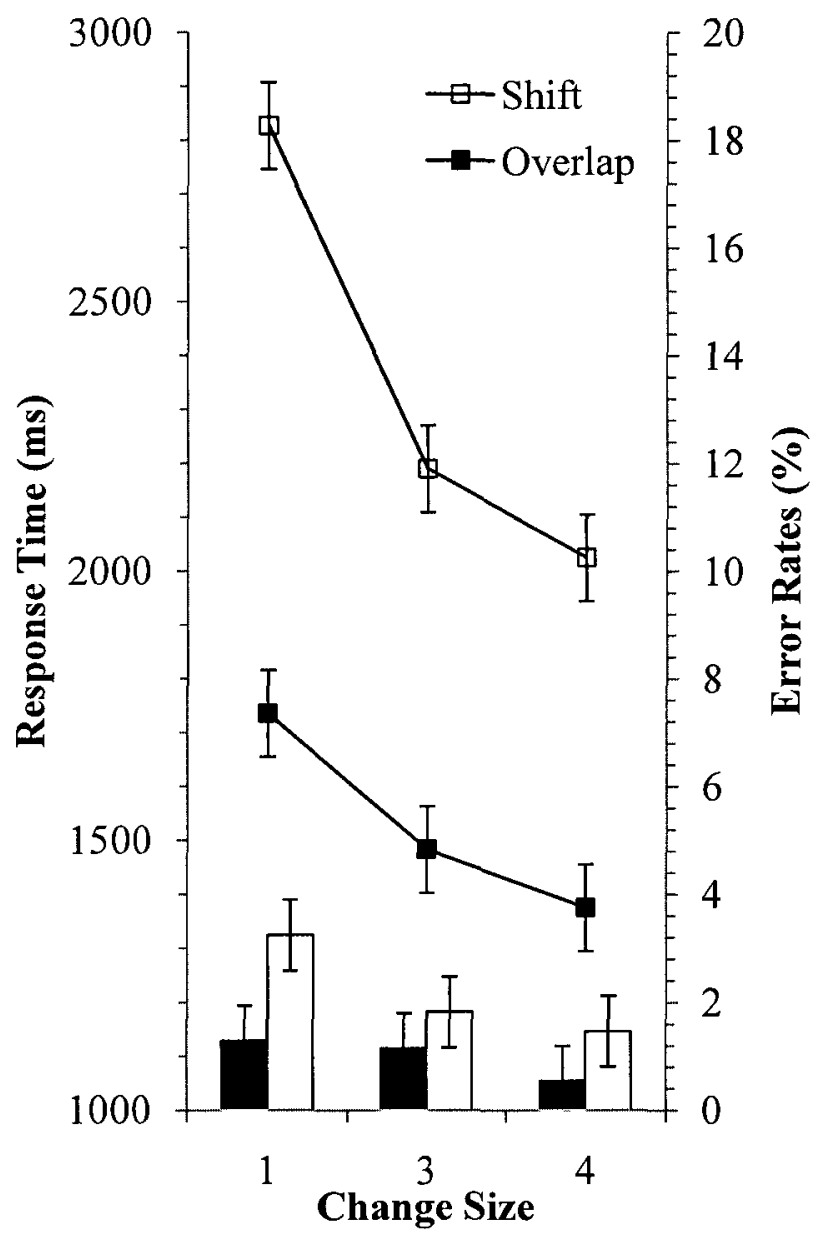

Figure 3. Experiment 1. Mean reaction times and error rates as a function of Display Type and Change Size with $95 \%$ confidence intervals (Jarmasz \& Hollands, 2009) 


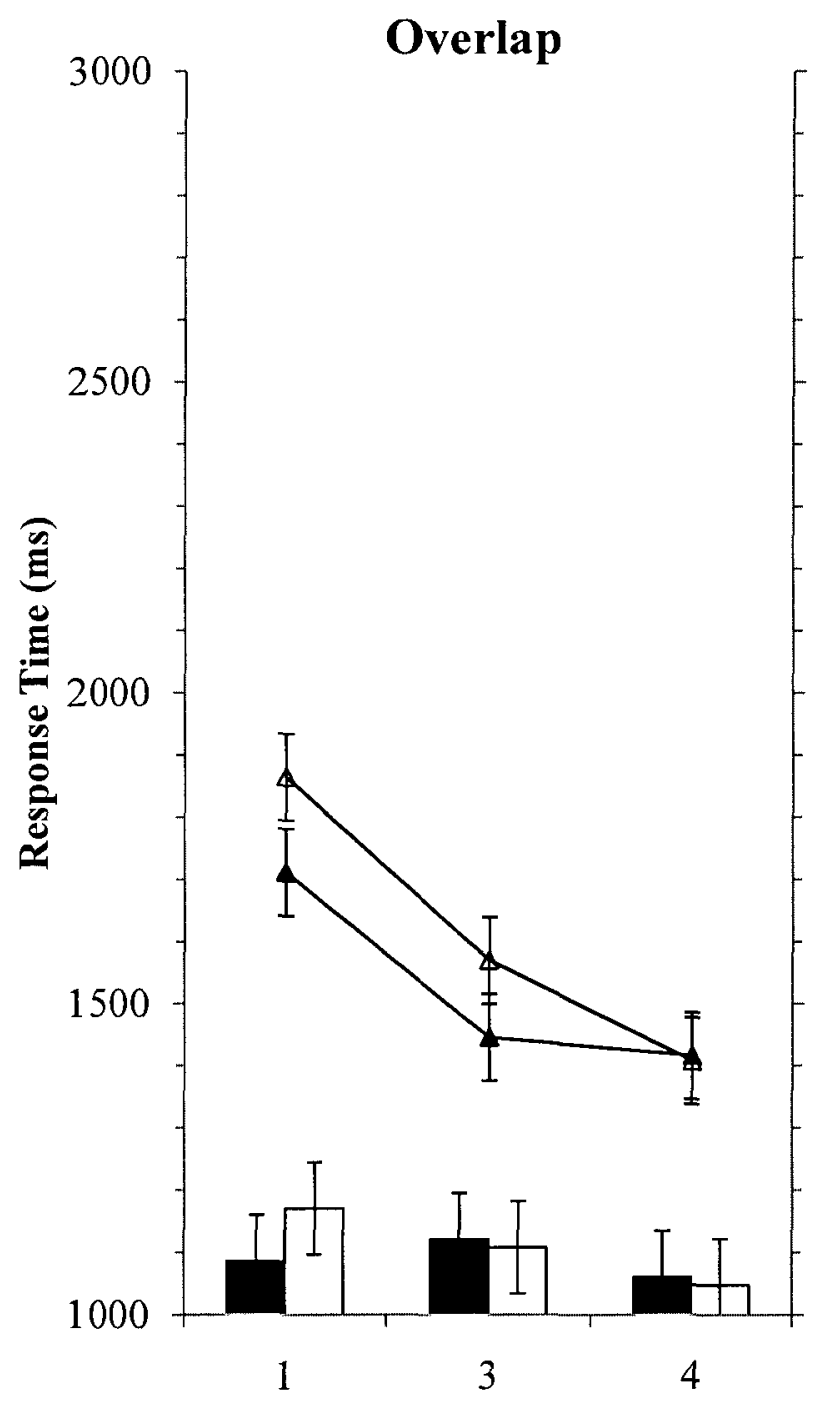

Change Size

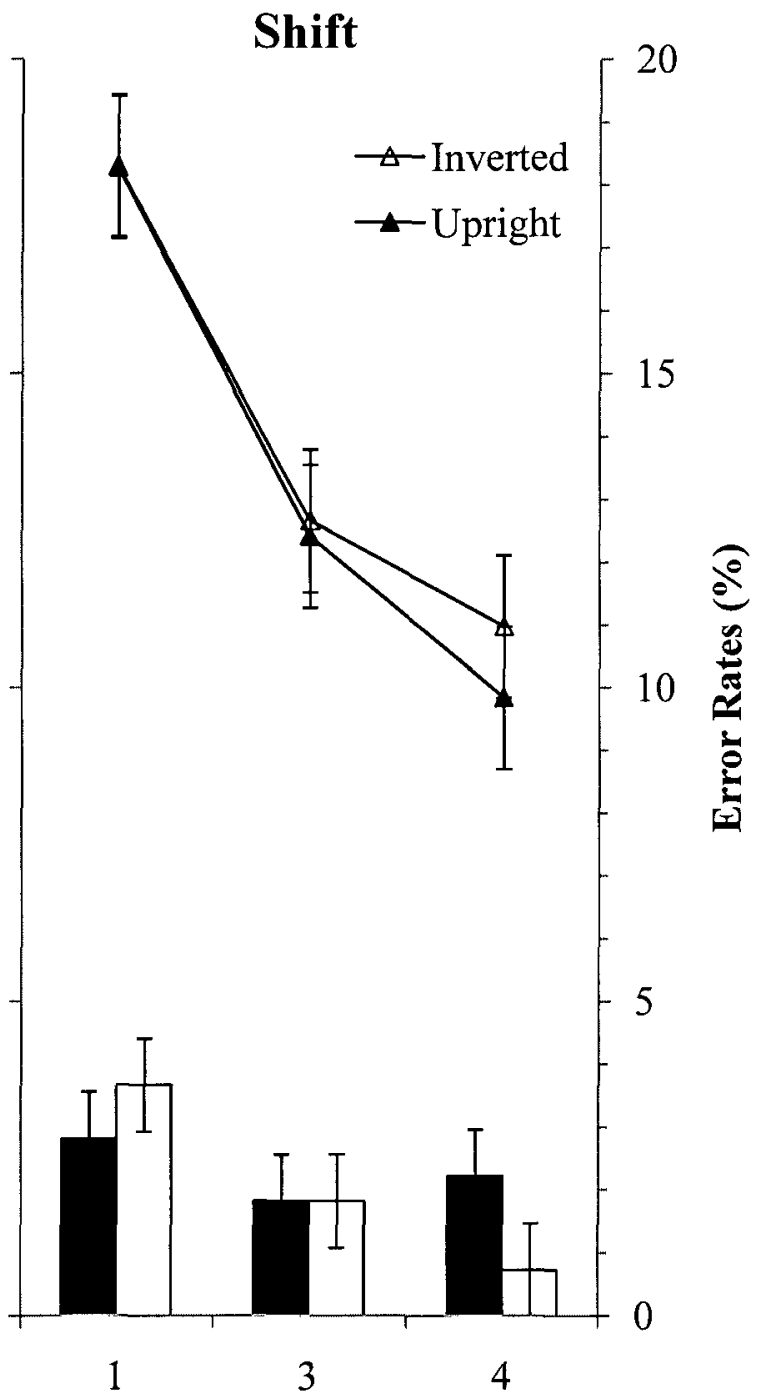

Change Size

Figure 4. Experiment 1. Mean reaction times and error rates as a function of Orientation and Change Size for each Display Type with $95 \%$ confidence intervals (Jarmasz \& Hollands, 2009). Confidence intervals were computed using the MSEs from the 2 (Orientation: Upright vs. Inverted) by 3 (Change Size: 1 vs. 3 vs. 4 features) ANOVAs for each level of Display Type.

Error rates: Accuracy data were analyzed using the same ANOVA used for the RT data. There was a significant main effect of Display Type, $F(1,34)=16.17, \mathrm{MSE}=.001, \mathrm{p}<.001$, partial $\eta^{2}=322$. Participants made fewer errors in the Overlap condition $(1.0 \%)$ than in the Shift condition $(2.2 \%)$. The main effect of Change Size was significant $F(2,68)=6.13$, MSE $=.001$, 
$p=.004$, partial $\eta^{2}=.153$. Error rates decreased as Change Size increased from 1-feature (2.3\%) to 3 -features $(1.5 \%)$ to 4 -features $(1.0 \%)$. Orientation did not significantly affect error rates $(\mathrm{F}<$ 1).

The interaction between Display Type and Change Size was marginally significant $F(2$, $68)=2.2, \operatorname{MSE}=.001, p=.061$ (see Figure 3). The effect of Change Size was larger in the Shift condition than in the Overlap condition. These data are consistent with the RT data, thus there is no evidence for a speed-accuracy trade-off. The interaction between Orientation and Change Size was marginally significant, $F(2,68)=2.40, \mathrm{MSE}=.001, p=.066$. There was a larger effect of Change Size for inverted letters than for upright letters. No other interactions were significant $(\mathrm{Fs}<1.2)$.

\section{Discussion}

The finding that changes were harder to detect in the Shift condition than the Overlap condition replicates previous work where change detection performance was compared across Overlap and Shift conditions (Irwin, 1991; Phillips, 1974; Shore \& Klein, 2000; Williams \& Simons, 2000). The effects of Change Size and Orientation in the Overlap condition is consistent with those of Tovey (2011), whose experimental parameters were identical to the Overlap condition in the present study (except that a 4-feature change was included here).

The Display Type manipulation had a marked effect on change detection performance. On average, participants took $804 \mathrm{~ms}$ longer to detect a change when $\mathrm{A}$ and $\mathrm{A}^{1}$ appeared in different locations compared to when they appeared in the same location. An inspection of Figure 3 reveals that the Change Size effect was markedly larger in the Shift condition than the Overlap condition. The larger effect of Change Size in the Shift condition suggests that without bottom-up information about $\mathrm{A}$ and $\mathrm{A}^{1}$ available in iconic memory, participants detected changes 
based on whatever amount of featural information they were able store in short-term memory within the $280 \mathrm{~ms}$ timeframe of the appearance of an array and an ISI.

The fact that the effect of Orientation was not differentially affected by Display Type (i.e., no Display Type $\mathrm{x}$ Orientation interaction) indicates that participants did not rely more heavily on top-down information about $\mathrm{A}$ and $\mathrm{A}^{1}$ to compensate for the lower amount of bottomup information available for comparison the Shift condition. Rather, the effect of Orientation was actually smaller in the Shift condition than the Overlap condition. While the interaction between Display Type and Orientation was non-significant, a post hoc comparison examining the simple effects of Orientation across Display Type revealed a significant effect of Orientation in the Overlap condition, but not in the Shift condition. The non-significant effect of Orientation in the Shift condition could indicate that this small shift of spatial attention (approximately 1 degree) interfered with the consolidation of information into STM to the extent that top-down knowledge of familiar (upright) letters was not able to exert an influence. The parameters used in the Shift condition may have forced participants begin to shift their attention from the location of $A$ to the location of $\mathrm{A}^{1}$ before $\mathrm{A}$ disappeared from the screen. This would mean that participants had less time to consolidate representations of the letters in the Shift condition than in the Overlap condition, and were thus more likely to rely on bottom-up (featural) information to detect the change. This would be expected to result in a larger effect of Change Size and smaller effect of Orientation in the Shift condition, which is exactly what was observed. However, the failure to find an effect of Orientation in the Shift condition could also be attributable to a lack of power (power $=.03$ ), especially given that the Orientation by Display Type interaction was not significant. 
To sum, Experiment 1 showed that at an $80 \mathrm{~ms}$ ISI, it is substantially more difficult to detect changes when $A$ and $\mathrm{A}^{1}$ appear in different locations than when they overlap spatially. Change detection performance in the Shift condition consistent with the notion that participants compared $\mathrm{A}$ and $\mathrm{A}^{1}$ based on relatively sparse featural representations that were not affected by top-down information related to $\mathrm{A}$ and $\mathrm{A}^{1}$.

While participants did not use top-down information about $\mathrm{A}$ and $\mathrm{A}^{1}$ to compensate for the greater difficulty of the Shift condition in Experiment 1, it is possible that by increasing the ISI will allow for a greater role of top-down information in the Shift condition, if the Shift condition is still more difficult with a longer ISI. A longer ISI will give participants more time to consolidate information form $\mathrm{A}$ and $\mathrm{A}^{1}$ into more robust representation in STM, which Richards (2002) has shown results in a greater role of top-down information. Therefore, Experiment 2 increased the ISI to $500 \mathrm{~ms}$.

\section{Experiment 2}

The objective of Experiment 2 was to examine the effect that a spatial manipulation has on change detection when $\mathrm{A}$ and $\mathrm{A}^{1}$ are separated by a $500 \mathrm{~ms}$ ISI. Experiment 1 showed that performance on a change detection task was substantially worse when $A$ and $A^{1}$ had to be compared in different spatial locations with an $80 \mathrm{~ms}$ ISI. The larger effect of Change Size in Experiment 1's Shift condition suggests that comparisons were more likely to be based on sparse featural information held in STM within the $280 \mathrm{~ms}$ SOA between A and $\mathrm{A}^{1}$ than when the two displays appeared in the same location. The non-significant interaction between Display Type and Orientation further indicated that top-down information did not facilitate change detection with an $80 \mathrm{~ms}$ ISI. 
The main reason why Experiment 1 yielded a large effect of Display Type is likely because participants could compare $\mathrm{A}$ and $\mathrm{A}^{1}$ based on quickly-stored bottom-up information held in iconic memory in the Overlap condition, but not in the Shift condition (Phillips, 1974; Irwin, 1991; 1992). Therefore, increasing the ISI to $500 \mathrm{~ms}$, which is beyond the duration of iconic memory, should reduce the effect of Display Type, as information stored in iconic memory will not greatly facilitate performance in the Overlap condition. Further, Shore and Klein (2000) provided evidence that it takes more time to encode $\mathrm{A}$ and $\mathrm{A}^{1}$ when they appear in different spatial locations. This implies that people require relatively well-consolidated representations in order efficiently compare $\mathrm{A}$ and $\mathrm{A}^{1}$ when they do not overlap. If stronger representations are required to detect changes across different spatial locations, and if all that is required to strengthen these representations is time, then increasing the SOA between $\mathrm{A}$ and $\mathrm{A}^{1}$ to $700 \mathrm{~ms}$ should reduce the size of the Display Type effect. Other studies (Brockmole \& Irwin, 2005; Williams \& Simons, 2000) provide evidence that with ISIs beyond the duration of iconic memory, it can still be more difficult to retain a detailed representation of the visual aspects of stimuli across a spatial shift in order to detect a change than to hold that representation in the same spatial location. It is possible that top-down information may play a greater role when detecting changes in non-overlapping objects with a $500 \mathrm{~ms}$ ISI than with the $80 \mathrm{~ms}$ ISI in Experiment 1. This top-down information may facilitate retention of visual information across a spatial shift, or it may facilitate the comparison of non-overlapping stimuli on non-visual dimensions.

\section{Predictions}

If spatial location does not affect representations stored in short-term memory, then the effects of Change Size and Orientation should be equal across the Overlap and Shift conditions, 
because there would be no benefit to comparing $\mathrm{A}$ and $\mathrm{A}^{1}$ in the same location versus different locations. Conversely, a greater difficulty of retaining visual representations in short-term memory across a spatial shift could be reflected in two different patterns of interactions between Display Type and Change Size, and Display Type and Orientation. If it is more difficult to compare non-overlapping objects than overlapping objects based on information stored in STM, and if top-down information cannot be used to compensate for this greater difficulty, then the effect of Change Size should be larger in the Shift condition than the Overlap condition (Display Type and Change Size should interact), whereas the effect of Orientation should be equal in the Overlap and Shift conditions (Display Type and Orientation should not interact). Conversely, if it is more difficult to compare non-overlapping objects than overlapping objects based on information stored in STM, and if top-down information can be used to compensate for this greater difficulty, then the effect of Orientation should be larger in the Shift condition than the Overlap condition (Display Type and Orientation should interact). Top-down information could facilitate performance in the Shift condition in two ways: First, top-down information about upright letters may better allow participants to recreate and 'clean-up' visual representations stored in short-term memory after shifting attention to a new location. Conversely, participants may encode $\mathrm{A}$ and $\mathrm{A}^{\mathrm{1}}$ on non-featural dimensions (e.g., verbal codes, categories). The key difference between whether Orientation facilitates performance in the Shift condition by helping to 'clean up' visual representations or by allowing for representations on non-featural dimensions is that the latter should result in a smaller effect of Change Size in the Shift condition than in the Overlap condition.

Cross-experiment effects: It is hypothesized that the $500 \mathrm{~ms}$ ISI would result in a smaller performance decrement in the Shift condition in Experiment 2 than the Shift condition in 
Experiment 1. This would be evidenced by an interaction between Experiments (1 vs. 2) and Display Type.

The Change Size effect was expected to be smaller in Experiment 2 than Experiment 1, as per Richards (2002) finding that the Change Size effect decreases at longer ISI lengths.

It was expected that factors influenced by top down information about $\mathrm{A}$ and $\mathrm{A}^{1}$ would have a more prominent effect in Experiment 2 than Experiment 1, based on Richards' (2002) finding that familiarity (a top-down factor) has larger effects as ISI increases. Therefore, the effect of Orientation, which is a proxy for familiarity, was expected to be larger in Experiment 2 than in Experiment 1.

\section{Method}

Experiment 2 was identical to Experiment 1 with the exception that the ISI was increased to $500 \mathrm{~ms}$.

Participants: Twenty-five Carleton University undergraduate students participated for 1 $\%$ course credit.

\section{Results}

Response times: Response times for correct trials were submitted to the same recursive outlier removal procedure used in Experiment 1. This resulted in $1.78 \%$ of the trials being excluded. The remaining data were analyzed using a 2 (Display Type: Overlap vs. Shift) by 3 (Change Size: 1 vs. 3 vs. 4 features) by 2 (Orientation: Upright vs. Inverted) repeated measures ANOVA.

There was a significant main effect of Display Type, $F(1,24)=5.1, \mathrm{MSE}=849656, p=$ .033 , partial $\eta 2=.176$, where participants detected changes more quickly in the Overlap condition (3018 ms) than in the Shift condition (3260 ms). The main effect of Change Size was 
significant $F(2,48)=15.7, \mathrm{MSE}=1262654, p<.001$, partial $\eta^{2}=.395$. RTs decreased as Change Size increased from 1-feature (3362 ms) to 3-features (3114 ms) to 4-features (2945 ms). The main effect of Orientation was also significant, $F(1,24)=9.8, \mathrm{MSE}=500453, p=.005$, partial $\eta^{2}=.289$. Participants detected changes in upright letters (3002 ms) more quickly than inverted letters (3276 ms).

The interaction between Display Type and Orientation was significant, $F(1,24)=4.8$, MSE $=129648, p<.039$, partial $\eta^{2}=.165$ (see Figure 6). The effect of Orientation was larger in the Shift condition than the Overlap condition. None of the other interactions were significant $(\mathrm{Fs}<1)$.

Experiment 1 vs. Experiment 2: Three $2 \times 2$ mixed ANOVAs were conducted to assess the differences in the effects of Display Type, Orientation and Change Size across Experiments 1 and 2. A 2 (Display Type: Overlap vs. Shift) $\mathrm{x} 2$ (Experiment 1 vs. 2) ANOVA revealed that the effect of Display Type was smaller in Experiment 2 than in Experiment 1, $F(1,58)=20.2$, MSE $=113423, p<.001$. A 2 (Orientation: Upright vs. Inverted) $\times 2$ (Experiment 1 vs. 2) ANOVA revealed that the effect of Orientation was larger in Experiment 2 than Experiment 1, $F(1,58)=$ $6.0, \mathrm{MSE}=44665, p=.018$. A 3 (Change Size: 1 vs. 3 vs. 4 features) $\times 2$ (Experiment 1 vs. 2 ) ANOVA revealed that the effect of Change Size was smaller in Experiment 2 than Experiment 1, $F(2,116)=3.7, \operatorname{MSE}=46040, p=.027$. 


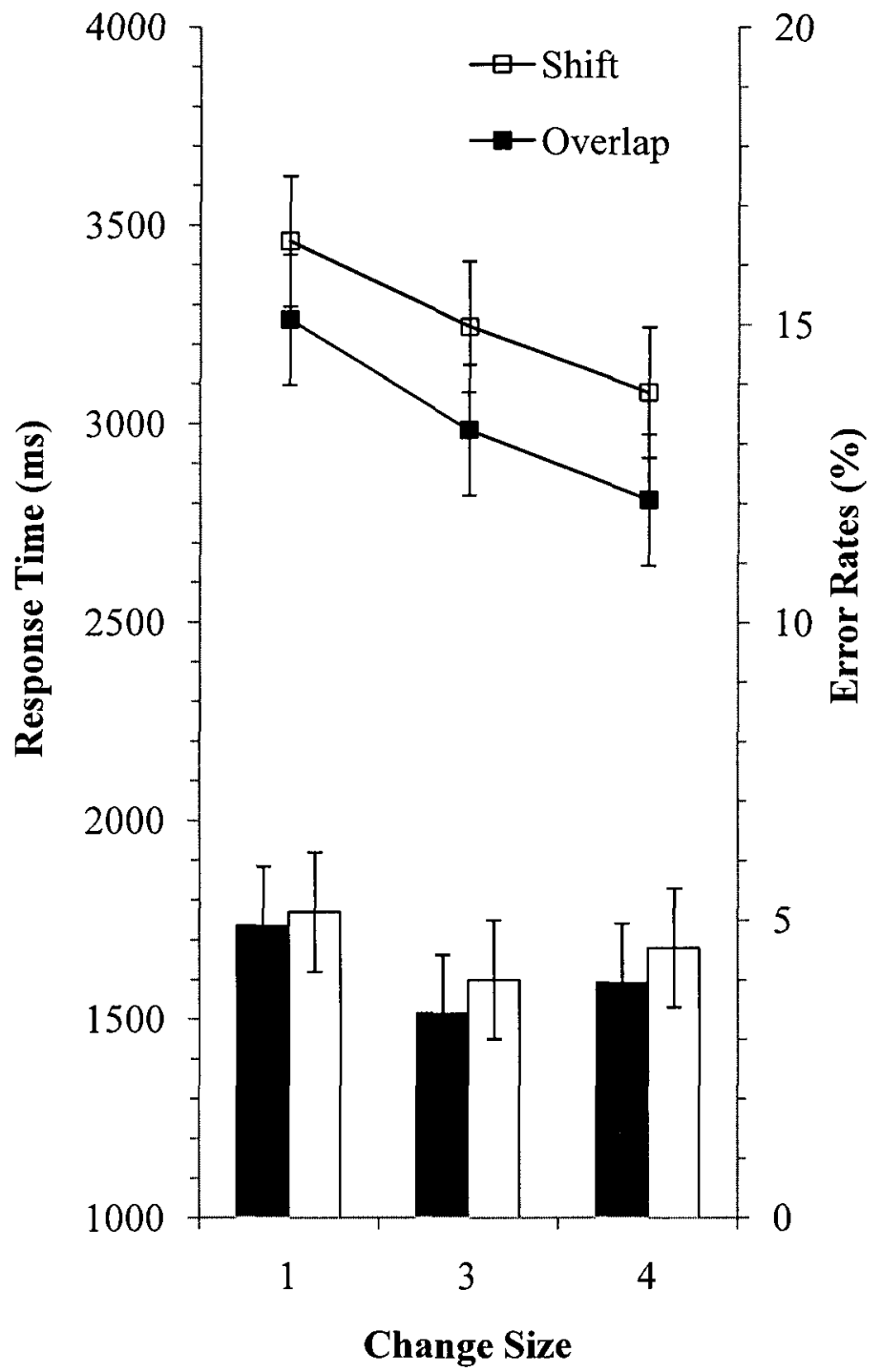

Figure 5. Experiment 2. Mean reaction times and error rates as a function of Display Type and Change Size with $95 \%$ confidence intervals. 


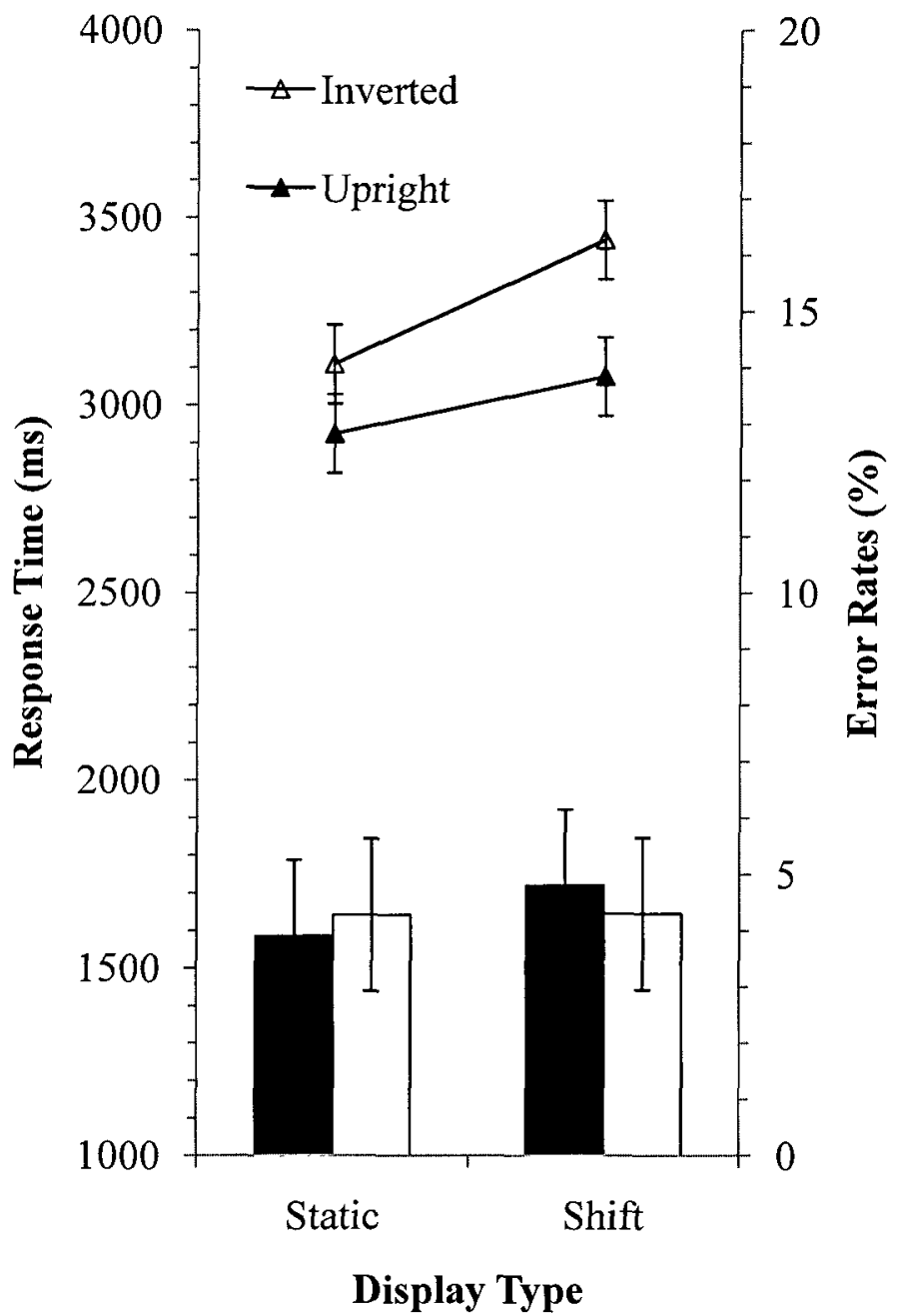

Figure 6. Experiment 2. Mean reaction times and error rates as a function of Display Type and Orientation with $95 \%$ confidence intervals. 

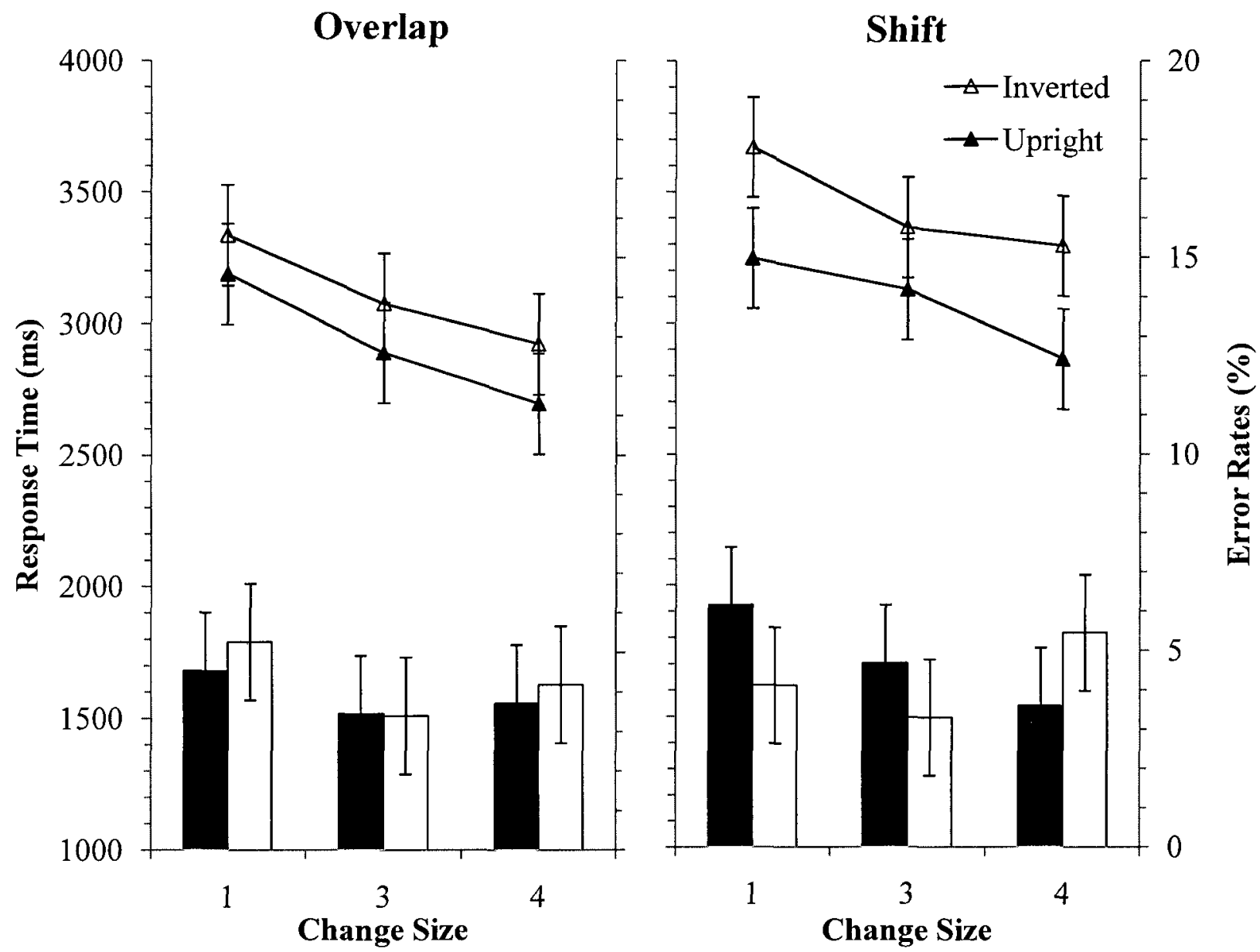

Figure 7. Experiment 2. Mean reaction times and error rates as a function of Orientation and Change Size for each level of Display Type with $95 \%$ confidence intervals.

Error rates: Accuracy data were analyzed using the same ANOVA used for the RT data. The main effect of Change Size was significant $F(2,48)=4.0, \mathrm{MSE}=.001, p=.026$, partial $\eta^{2}$ $=.142$, where error rates decreased as Change Size were higher for 1 -feature $(5.0 \%)$ than for 3 feature $(3.7 \%)$ and 4 -feature $(4.3 \%)$ changes. No other main effects or interactions were significant $(\mathrm{F} \mathrm{s}<1.6)$. 


\section{Discussion}

The fact that the effect of Orientation was larger in the Shift condition than the Overlap condition suggests that top-down information played a larger role in the Shift condition than the Overlap condition. Taken with the fact that changes in the Overlap condition were detected more quickly than in the Shift condition, the greater role of top-down information in the Shift condition provides evidence that it is more difficult to shift a representation stored in short-term memory to a new spatial location than to hold it in the same spatial location. A key question for Experiment 2 was whether participants would be more likely to compare $\mathrm{A}$ and $\mathrm{A}^{1}$ on non-visual dimensions in the Shift condition than in the Overlap condition. The lack of a significant interaction between Display Type and Change Size is not consistent with the hypothesis that participants were more likely to compare letters on non-visual dimensions in the Shift condition than in the Overlap. Rather, an inspection of the Figure 5 indicates that participants ultimately compared letters based on the same visual characteristics regardless of whether they shifted. Therefore, given the significant Display Type by Orientation interaction coupled with the nonsignificant interaction between Display Type and Change Size, it seems likely that the role of top-down information in the Shift condition was to help participants retain a visual representation of array A in short-term memory that could be compared with the subsequent visual precept of $\mathrm{A}^{1}$.

The smaller effect of Change Size and larger effect of Orientation in Experiment 2 than Experiment 1 confirmed that the $500 \mathrm{~ms}$ ISI allowed participants to consolidate higher-level representations of $\mathrm{A}$ and $\mathrm{A}^{1}$. This, combined with the smaller effect of Display Type in Experiment 2 confirms the hypothesis that higher-level representations allow for more efficient comparisons of visual information in different spatial locations. Other across-experiment effects 
of interest are the differential effects that ISI length had on how the Change Size and Orientation manipulations differed in Experiments 1 and 2.

In sum, the results of Experiment 2 indicate that it is more difficult to detect a change across spatial locations when the ISI between $\mathrm{A}$ and $\mathrm{A}^{1}$ is beyond the duration of iconic memory, and comparisons must be made based on information stored in STM. The larger effect of Orientation in the Shift condition than the Overlap condition indicates a greater difficulty of shifting a visual representation stored in short-term memory to a different location than holding that representation in the same location. Top-down information seemed to alleviate the difficulty of shifting representations to a new spatial location by allowing for better retention of the visual characteristics of letters, because the effect of Display Type was smaller for upright letters than for inverted letter, whereas there was no substantial difference in the effects of Change Size in the Overlap and Shift conditions. However, the slightly smaller effect of Change Size and smaller amount of variance accounted for by Change Size in the Shift condition does provide some evidence that participants compared $\mathrm{A}$ and $\mathrm{A}^{1}$ based on non-visual dimensions at least some of the time in Experiment 2.

\section{General Discussion}

The goal of this thesis was to compare how changes are detected when objects appear in different locations versus the same location. The general results can be summarized as follows: First, changes were easier to detect when objects appeared in the same location than if objects appeared in different locations. Second, the effect of whether objects appeared in the same or different locations was different depending on whether the temporal separation was short ( $80 \mathrm{~ms})$ or long $(500 \mathrm{~ms})$. The performance cost to having objects appear in different locations relative to the same location was markedly larger when the ISI was $80 \mathrm{~ms}(816 \mathrm{~ms}$ difference) relative to 
when the ISI was $500 \mathrm{~ms}$ ( $243 \mathrm{~ms}$ difference). This pattern suggests that the advantage to having objects appear in the same location versus different locations is substantially greater if the temporal separation is sufficiently brief to allow for comparisons to be made with information stored in iconic memory.

Furthermore, the advantage of the greater familiarity of upright versus inverted letters was larger for non-overlapping than overlapping displays with a long temporal separation, whereas the advantage of upright versus inverted letters was similar for non-overlapping and overlapping displays with a short temporal separation. This pattern suggests that while top-down information can be recruited to compensate for a greater difficulty of comparing non-overlapping letters, a strong enough object-based representation of the memorized display must first be consolidated in STM. If sufficient time is not available to create an object-based representation, then more top-down information cannot be recruited.

\section{Interpreting the Experiment by Display Type Interaction}

Previous research has shown a clear effect of spatial location on change detection when ISIs were within the duration of iconic memory. However, findings from Irwin (1991) and Phillips (1974) conflicted with those of Williams and Simons (2000) and Brockmole and Irwin (2005) as to whether spatial location plays a substantial role in detecting changes based on information stored in STM. The findings presented in this thesis support the notion that the spatial locations of objects plays a role in detecting changes based on information stored in STM and is therefore consistent with Williams and Simons, and Brockmole and Irwin.

There are two possible reasons why this thesis and Williams and Simons (2000) showed an effect of spatial location in change detection using STM-based comparisons while Irwin (1991) and Phillips (1974) did not. First, the size of the spatial shift differed between all the 
studies. The spatial shifts in Phillips and Irwin were .5 and .8 degrees in visual angle, respectively. The spatial shift in this thesis was approximately 1 degree in visual angle and the occluding box in Williams and Simons was 7 degrees in visual angle. The spatial shift used in this thesis is not much larger than the .5 and .8 degrees used in Phillips and Irwin, however, it is important to note that the spatial shift in Phillips and Irwin comprised one cell-width of a dot matrix, which means that a large portion of their $\mathrm{A}$ and $\mathrm{A}^{1}$ displays overlapped in their spatial shift condition. Conversely, the spatial shifts in this thesis (and in Williams and Simons) were sufficiently large such that no part of the $A$ and $A^{1}$ objects appeared in the same spatial locations. It is possible that the effect that a spatial shift can have on STM-based comparisons is related to the size of the spatial shift relative to the size of the objects, rather than being related to the absolute size of the shift. If a shift is small enough such that $\mathrm{A}^{1}$ occupies some of the area previously occupied by A, then the shift may be perceived as if A had moved. In contrast, if A and $\mathrm{A}^{1}$ appear in completely different locations, then they may be perceived as distinct objects. The perception of a single object moving versus two distinct objects appearing in different locations may change the task such that the latter in substantially more difficult than the former.

A second difference between (1) Phillips' and Irwin's studies and (2) this thesis and Williams and Simons is that the former used dot patterns whereas the latter used letters and geons. Retaining a dot pattern in STM may place more emphasis on spatial locations, whereas retaining a letter or collection of geons in STM places more emphasis on retaining the visual characteristics of objects. One might suggest that STM for spatial information is less affected by a spatial shift than memory for visual characteristics. However, this is unlikely as STM for spatial locations has been shown to be susceptible to shifts of attention (Awh, Jonines \& Reuter- 
Lorenz, 1998). Further, Brockmole and Irwin (2005) also showed that a 20 degree spatial shift affects performance on a dot-pattern comparison task.

While a location effect was observed with temporal separations both within (Experiment 1) and beyond (Experiment 2) the duration of iconic memory, this effect was markedly larger in Experiment 1 than Experiment 2. The larger location effect in Experiment 1 is consistent with Irwin's and Phillips' assertion that iconic memory is strongly (if not absolutely) tied to spatial location. It is likely that when the overlapping displays were separated by $80 \mathrm{~ms}$, participants were able to make comparisons largely based on bottom-up information stored in iconic memory, whereas when displays shifted, they made comparisons based on representations created in STM within the $280 \mathrm{~ms}$ SOA between A and $\mathrm{A}^{1}$.

\section{Spatial Location and Iconic Memory}

The location effect interacted with Change size in Experiment 1, in that the number of features changing had a significantly larger effect on change detection when displays appeared in different locations than when they appeared in the same location. This interaction indicates that participants had access to more (or better quality) featural information the displays in the overlap condition than in the non-overlap condition. This enhanced representation of featural information in the overlap condition supports the more general assertion that information stored in iconic memory is particularly useful when temporally separated displays appear in the same location.

If shifting visual information precludes the use of bottom-up information stored in iconic memory to make comparisons, then it might be expected that other types of information (e.g., top-down) information would be relied on to detect changes in displays that do not overlap spatially. Indeed, Shore and Klein (2000) provided evidence that people rely on higher level attributes of scenes when comparing images across different locations than when images 
appeared in the same location. However, the fact that familiarity (a high-level attribute) did not interact with Display Type in Experiment 1 does not support this claim. If higher level attributes are relied on more heavily to detect changes in displays that shift, then the effect of familiarity should have been larger in the shift condition than in the overlap condition. Rather, it is possible that this reliance on higher level attributes in shifting displays reported by Shore and Klein was caused by longer dwell times on the images and not the fact that the images shifted. This alternate explanation corresponds with the present findings that when the ISI was lengthened to $500 \mathrm{~ms}$ in Experiment 2, participants' responses exhibited a greater reliance on top-down information about the displays in both the Overlap and Shift conditions.

\section{Spatial Location and Short-Term Memory}

Experiment 2 showed that it is harder to use information stored in STM to detect changes in displays that appear in different locations than when they that overlap. The interaction between Display Type and Orientation indicates that top-down information (familiarity) has a larger effect on change detection when displays appear in different locations than when they appear in the same location. A key question in Experiment 2 was whether a greater reliance on top-down information in change detection for shifting displays was commensurate with the difficulty of comparing objects based purely on their featural characteristics. If non-overlapping displays were compared based on categorical or verbal information (e.g., a letter's name or sound), then the number of features changing should have had a smaller effect on performance when the displays shifted than when they overlapped. Interestingly, the change size effect did not differ depending on whether displays appeared in the same or different locations. Therefore, the greater reliance on top-down information for detecting changes in shifting displays did not seem to be primarily related to the difficulty with which featural information was used to detect 
changes. Rather, the additivity between Change Size and Display Type indicates that participants compared objects based on the same visual characteristics regardless of whether they appeared in the same location or different locations. In this view, the greater import of top-down information for detecting changes in non-overlapping objects in Experiment 2 may indicate that it is more difficult to retain visual characteristics of objects across a spatial shift compared to when objects remain at the same location. Prior knowledge about objects stored in long-term memory may act as a cue in either retaining or reconstructing the visual characteristics of objects after they have undergone a spatial shift. Williams and Simons (2000) and Brockmole and Irwin (2005) have provided evidence that it is more difficult to shift a one-to-one visual representation of an object stored in STM to a new location than it is to hold it in the same location. The results of this thesis, therefore, support the view that shifting an object's location makes it more difficult to retain STM representations of those objects. However, this difficulty is alleviated, in part, by relying on top-down information (prior knowledge) to assist with the reconstruction of the STM representations.

Interestingly, the Display Type x Familiarity interaction observed in Experiment 2 when the ISI was $500 \mathrm{~ms}$ was not seen in Experiment 1 where the ISI was $80 \mathrm{~ms}$. Even though the comparisons made in the Overlap condition in Experiment 1 were probably largely made in iconic memory, the comparisons in the Shift condition would have been made in STM. Thus, familiarity should have had an effect in the Shift condition in Experiment 1 (but not in the overlap condition). It is therefore surprising that Familiarity did not interact with Display Type. The reason for this discrepancy between Experiments 1 and 2 may simply be a matter of how long participants had to consolidate the displays into robust STM representations. Richards (2002) and this thesis showed that top-down information plays a larger role as the SOA between 
two displays increases. Richards' attributed this effect to the fact that it takes time to consolidate visual information into object-based representations in STM that can benefit from pre-existing information in long-term memory. Therefore, it is possible that the $280 \mathrm{~ms} \mathrm{SOA}$ in Experiment 1 was not long enough for participants to consolidate $A$ and $A^{1}$ into object-based representations that could benefit from top-down information (e.g., familiarity).

\section{Summary and Conclusion}

The findings presented in this thesis show that changes in objects that must be compared across different spatial locations are more difficult to detect than when objects remain stationary. If comparisons are made across a relatively short temporal interval ( $80 \mathrm{~ms}$ in this thesis), then detecting changes in shifting objects depends more heavily on the visual characteristics of the change than when detecting changes in overlapping objects. However, if enough time is available to create stable representations of objects in STM, then pre-existing information about those objects from long-term memory may alleviate some of the difficulty associated with detecting changes in objects in dynamic displays. The greater reliance on top-down information when detecting changes in non-overlapping displays suggests that the role that top-down information plays is greater when we detect changes in day-to-day life than when changes are detected in static displays on computer screens. Therefore, more consideration of factors that may affect higher level, top-down processing is warranted for future studies that attempt to generalize experimental findings to cognitive processing in the real-world.

In Experiment 1, a simple horizontal shift of one degree in visual angle increased the time it took to detect changes by as much as $50 \%$ and increased the impact that the size of the change had on change detection. Conversely, in Experiment 2 when the ISI was increased from $80 \mathrm{~ms}$ to $500 \mathrm{~ms}$, the spatial manipulation no longer affected the influence that change size had on 
performance, but changed the observer's reliance on top-down information to detect changes. The effects that shifting a display and increasing the time to consolidate information seen in these displays had on change detection serve as a reminder of how complex the relationship is between our mind and our environment. 


\section{References}

Awh, E., Jonides, J., \& Reuter-Lorenz, P. A., (1998). Rehearsal in Spatial Working Memory. Journal of Experimental Psychology, 24 (3), 780 - 790.

Brockmole, J. R., (2005). Eye movements and the integration of visual memory and visual perception. Perception \& Psychophysics, 67 (3), $495-512$.

Inquisit 3.05.0 [Computer software]. (2010). Seattle, WA: Millisecond Software LLC.

Irwin, D. E. (1991). Information integration across saccadic eye movements. Cognitive Psychology, 23, $420-456$.

Irwin, D. E. (1992). Memory for position and identity across eye movements. Journal of Experimental Psychology: Learning, Memory, and Cognition, 18 (2), $307-317$.

Jarmasz, J. \& Hollands, J. G. (2009). Confidence Intervals in Repeated-Measures Designs: The Number of Observations Principle. Canadian Journal of Experimental Psychology, $63(2), 124-138$.

Luck, S. J. \& Vogel, E. K. (1997). The capacity of visual working memory for features and conjunctions. Nature, 390, $279-281$.

Mitroff, S. R., Simons, D. J., \& Franconeri, S. L. (2002). The siren song of implicit change detection. Journal of Experimental Psychology: Human Perception and Performance, 28 (4), $798-815$.

Phillips, W. A. (1974). On the distinction between sensory storage and short-term visual memory. Perception \& Psychophysics, 16, $283-290$.

Rensink, R. A., O'Regan, J. K., \& Clark, J. J. (1997). To see or not to see: The need for attention to perceive changes in scenes. Psychological Science, 8, 368-373.

Rensink, R. A., O'Regan, J. K., \& Clark, J. J. (2000). On the failure to detect changes in scenes across brief interruptions. Visual Cognition, 7, 127-145.

Richards, E. (2002). The Shift from Feature-based to Object-based Representations in the Search for a Change: The Role of Short-Term Consolidation. Unpublished PhD thesis, University of Waterloo, Waterloo, Ontario, Canada. 
Shore, D. I., \& Klein, R. M. (2000). The effects of scene inversion on change blindness. Journal of General Psychology, 127, 27-43.

Stoltz, J.A. \& Joliceour, P. (2004) Changing features do not guide attention in change detection: Evidence from a spatial cuing paradigm. Psychonomic Bulletin \& Review, 11(5), 870-

Tovey, M. (2011). Seeing Changes: How Familiarity Alters Our Perception of Change. Unpublished $\mathrm{PhD}$ thesis. Carleton University, Ottawa, Ontario, Canada.

Van Selst, M., \& Jolicoeur, P. (1994). A Solution to the Effects of Sample Size on Outlier Elimination. Quarterly Journal of Experimental Psychology, 47A, 631-650.

Vogel, E. K., Woodman, G. F., \& Luck, S. J. (2001). Storage of Features, conjunctions and objects in visual working memory. Journal of Experimental Psychology: Human Perception and Performance, 27, $92-114$.

Williams, P. \& Simons, D. J. (2000). Detecting changes in novel, complex threedimensional objects. Visual Cognition, 7 (1/2/3), 297-322. 